\title{
Indagine geologica e geofisica su di un affioramento argilloso nei pressi di Pratica di Mare - Roma
}

\author{
(Geological and geophysical research on an outcrop of clay near \\ Pratica di Mare - Rome)
}

\author{
A. Sonaglia - E. Volponi $(*)$
}

Ricevuto il 30 Giugno 1972

Riassunto. - Nei pressi di Pratica di Mare ('Tav. Ardea - Fog. 158 IV N.O.) è stata eseguita una campagna di sondaggi elettrici i cui risultati, associati ai dati geologici di rilevamento e a dati reperiti dalla bibliografia geologica della zona (sondaggi meccanici, elettrici e gravimetrici), hanno permesso di porre in relazione la paleomorfologia ed il significato strutturale di un affioramento argilloso cupoliforme con i lineamenti tettonici prevulcanici della regione.

Summary. - In the area of Pratica di Mare (Tav. Ardea - Fog. 158 IV N.O.) a series of electrical soundings has been conducted. Its results, associated with data of geological surveys and those gathered by geological reports on the area (mechanical, electrical and gravimetrical soundings) allowed to establish links between paleomorphology and the structural meaning of a dome-shaped clay outcrop on the one hand and the prevolcanic tectonic features of the area on the other.

Nel corso del rilevamento geologico-tecnico della Tav. Ardea (Fog. 158 IV N.O.) in seala $1: 25.000$, attualmente in fase di esecuzione, è stato preso in particolare esame un affioramento argilloso nei pressi di Pratica di Mare, riportato dalla Carta Geologica d'Italia come Pliocene medio-superiore.

(*) Centro Studi per la Geologia Tecnica, C.N.R., Istituto di Geologia Applicata all'Ingegneria, Roma. 
Isaffioramento, venuto a giorno in seguito all'avanzamento del fronte di cava e allo sbancamento della copertura, costituita da sabbie e prorolti piroclastici, viene coltivato dalla "Soc. Iuaterizi Pratica di Mare "; il materiale, con opportuni procedimenti di lavorazione, risulta tra i migliori del genere per la produzione di laterizi per l'edilizia.

Ia cava is situata tra il paese di Pratica di Mare ed il Cimitero omonimo e precisamente sul lato sinistro del Fosso di Pratica (Fig. 1).

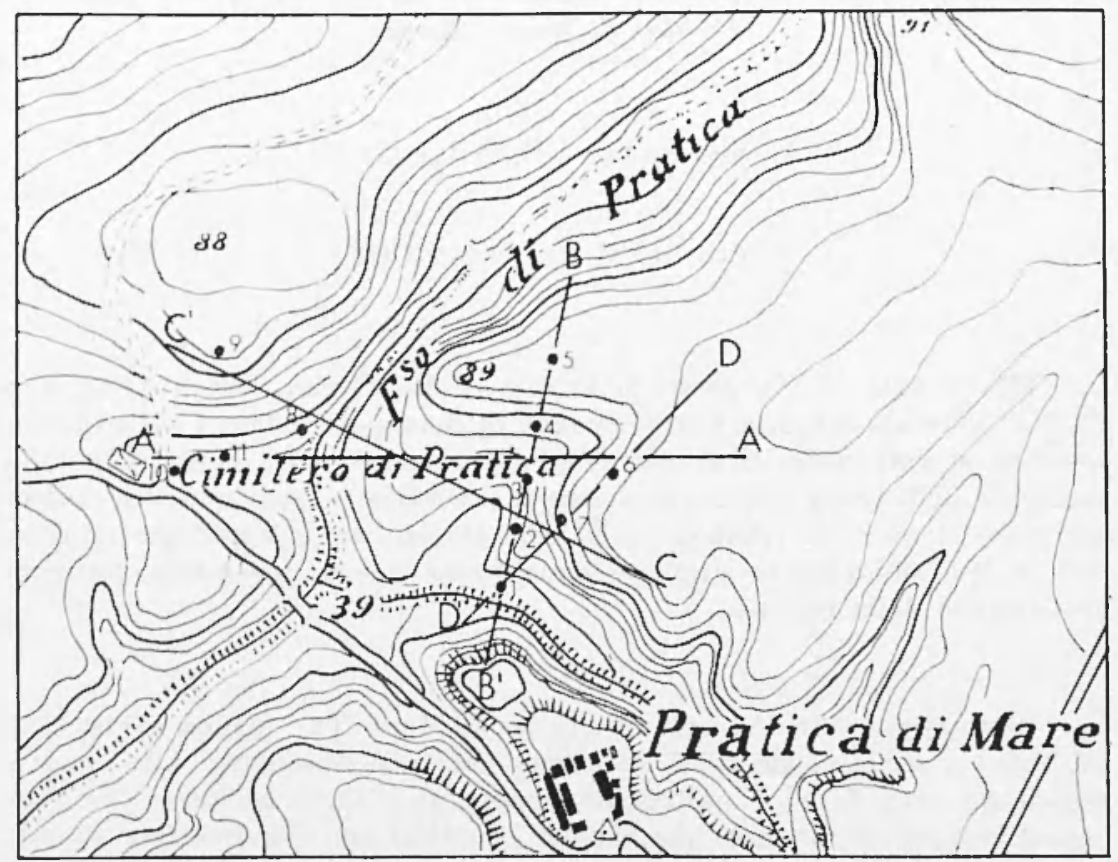

Figr. I - Planimetria e direttrici đei profili geoelettrici.

$\mathrm{B}^{\prime} \cdot-\cdot-\cdot-\mathrm{B}$, direttrice del profilo geoelettrico

- I. ..... ubicazione e numero dei profili geoelettrici

Ia zona in generale, presenta ma morfologia quasi pianeggiante con quote comprese tra i 70 ed i $90 \mathrm{~m}$ s.l.m. e viene attraversata da una incisione piuttosto profonda nella quale scorre il Fosso di Pratica; ad essa confluisce tutta ma serie di vie di deflusso alimentate esclusivamente nella stagione delle precipitazioni, di cui la più profonda risulta quella posta alla base del paese. I'affioramento argilloso, oggetto della presente nota, ̀̀ ubicato alla confluenza della incisione del Fosso di Pratica con quella posta alla base del paese. 
Sul fronte di cava si osserva una parete argillosa di circa 10 metri, costituita da un'alternanza di strati argillosi di colore grigio-azzurro di $40-50 \mathrm{~cm}$ di potenza, inclinati di $222^{\circ}-25^{\circ}$ con immersione $\times 30^{\circ} \mathrm{W}$, e intercalati da sottili livelli limoso-argillosi da poco coerenti a sciolti.

A tetto dell'affioramento si può osservare una paleo-superficie di erosione che taglia o segue l'andamento stesso della superficie di strato (fig. 2).
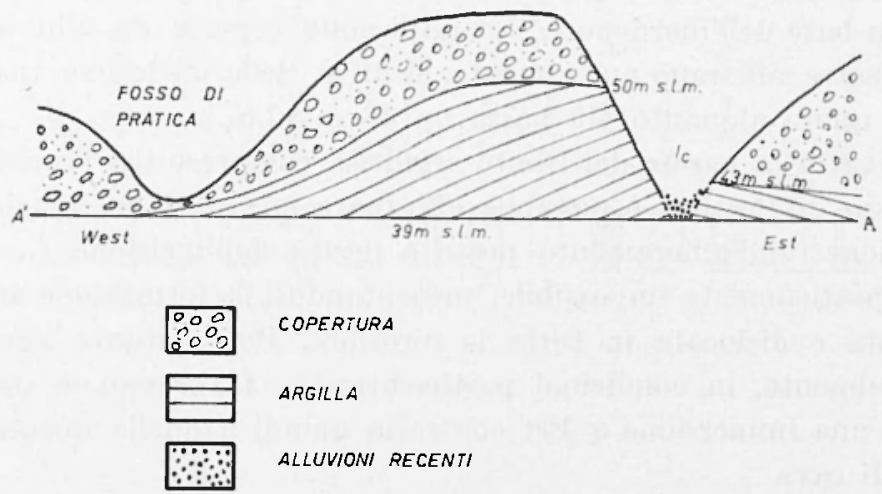

Fig. 2 - Sezione indicativa del fronte di cava.

La copertura del banco argilloso è costituita da sabbie continen tali e marine del tardo Quaternario $\left(^{*}\right)$, da tufi granulari grigi e pozzolane inferiori del vulcanismo laziale e da sabbie rosse eoliche ferrettizzate che coprono uniformemente i sottostanti materiali. La giacitura della copertura è sub-orizzontale e quindi in discordanza angolare con le argille sottostanti; nell'insieme la copertura ha una potenza complessiva di circa 25 metri.

Le prime osservazioni effettuate sul fronte di cava, disposto in direzione $\mathrm{EW}$, poste in relazione con l'ambiente geologico circostante, hanno fatto rilevare l'esistenza di determinati caratteri che possono dar luogo ad alcune interpretazioni nella ricostruzione degli eventi che hanno contribuito a determinare l'attuale situazione lito-stratigrafica.

(*) Le analisi micropaleontologiche sono state effettuate dal dott. M. Chiocelhini. 
Si rappresenta schematicamente la sezione del fronte di cara (fig. 2) indicato con la direttrice di profilo geoelettrico $A^{\prime}-A$ in fig. 1, in modo da rendere pin chiara l'esposizione dei dati ricavati.

Come indicato in figura il lato destro del fronte argilloso presenta, rispetto al lato sinistro, una altezza maggiore (50 $\mathrm{m}$ s.l.m.) e la stratificazione del banco argilloso segna una inclinazione regolare e costante per quasi tutta l'estensione dellaffioramento; avvicinandosi al Fosso di Pratica le argille cambiano rapirlamente la loro inclinazione assumenrlo una giacitura sub-orizzontale.

Alla base dell'incisione $I_{c}$, le argille sono coperte da alluvioni recenti mentre affiorano nuovamente, al di là della medesima incisione, arl una quota alquanto più bassa (q. $\$ 3 \mathrm{~m}$ s.l.m.).

Su tutta la parete del fronte argilloso, compreso tra l’incisione $I_{\text {: }}$ e il Fosso di Pratica, è possibile effettuare misure di inclinazione ed immersione; sullaffioramento posto a destra dell'incisione $I_{c}$, questo risulta praticamente impossibile, presentandosi la formazione argillosa fratturata e dislocata in tutte le direzioni. Puo soltanto aflermarsi che loculmente, in condizioni particolarmente favorevoli, es stata osservata una immersione a Est contraria quindi a quella misurata sul fronte di cava.

In base alle osservazioni effettuate sul luogo, si ritiene che l'affioramento sia il risultato di una modesta e locale tettonica che avrebbe interessato le argille di base, sollevandole e dislocandole dalla loro posizione originaria, prima della messa in posto dei materiali costituenti la copertura.

La presenza di una serie di faglie a piccolo rigetto, osservabili sul fronte di cava, interrotte al contatto tra le argille ed i materiali sovrastanti, e la giacitura degli strati del banco argilloso contraria sui due fianchi dell'incisione $I_{c}$, induce a ritenere possibile la presenza di una faglia, in direzione anti-appenninica, impostata lungo l'incisione $I_{c}$, il cui piano di scorrimento separerebbe l'affioramento su cui è impostato il fronte di cava da quello ribassato.

Sempre in base a osservazioni effettuate sul luogo i stato possibile ricavare considerazioni derlotte dai rapporti stratigrafici che legano le argille con la copertura, (Fig. 3).

Il contatto tra le argille plioceniche ed i depositi fluvio-lacustri continentali, ¿ marcato da una superficie di erosione; pertanto, i rapporti diretti tra le argille e le ghiaie-sabbiose sono da attribuire ar intensi fenomeni erosivi che hamno permesso tale contatto.

Gli esami paleontologici, effettuati su diversi campioni, hanno 


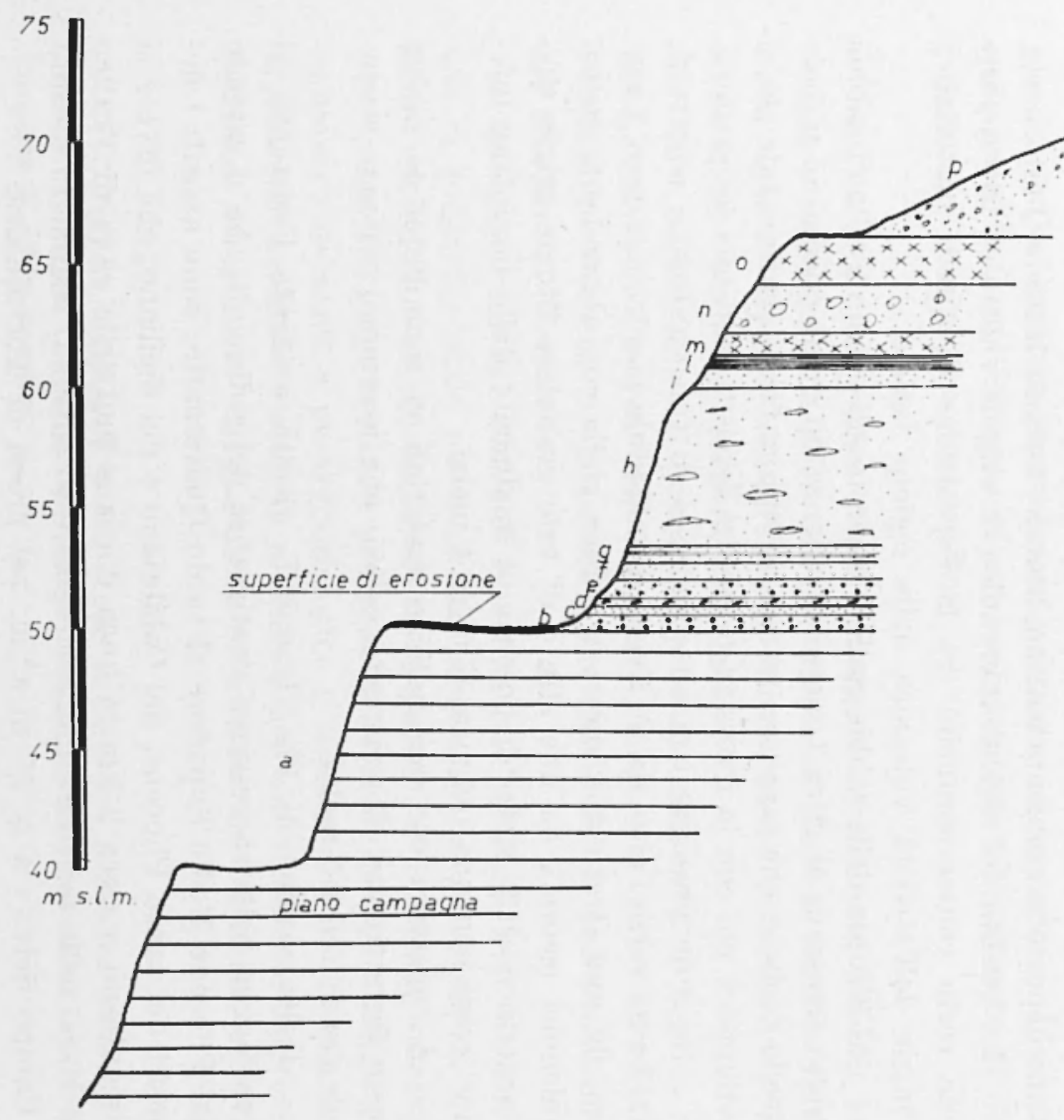

a Argille

$b$ ::: Ghiala sabb grigla o legg arrossata

c $\square$ Sabbia grigia

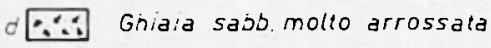

e $\therefore$ Sabbla con ghiala lievemente arrossata

$\square$ Sabbia grigio-giallo

$g$ Sabbia arrossata con livelleiti argiliosi

h- Sabbia gialla con macrofossiai e poupe

, $\square$ Sabbia mollo arrossata

Livello torboso

$m$ Materiaie grigio tufaceo

Po Sabbia gialla con inclusi vulcanici (scorie)

Turo antico

Q:: Pozzolane rosse

$9 \square$ Sabsie rosse

Fig. 3 - Sezione normale alla direttrice $A^{\prime} A$ del fronte di cava. 
permesso di datare le argille al Pliocene inferiore (zona a Globorotalia punticulata); lambiente di deposizione è tipicamente marino, neritico medio.

I depositi fluvio-lacustri a contatto e in discordanza con le argille sono costituiti da ma serie di livelli di ghiaia e sabbia della potenza complessiva di m 1,70, attribuibili probabilmente ad una fase continentale calda durante la quale si sono resi possibili i fenomeni di ossidazione che hanno conferito ai depositi stessi un grado di arrossamento più o meno intenso.

Sempre in concordanza stratigrafica è stato rilevato un altro orizzonte fossilifero, costituito da sabbie gialle a macrofossili, riferibile al tardo Quaternario. I macrofossili presenti, la scarsa quantità in microfossili, di regola in cattivo stato di conservazione, ed i resti organici più recenti non forniscono più sicure indicazioni cronostratigrafiche, trattandosi di termini diffusi dal Pliocene superiore al Quaternario. Vi sono, tuttavia, maggiori probabilita che tali sabbie si siano deposte in ambiente marino, litorale, durante il tardo Quaternario.

Isa qualità del residuo inorganico di origine vulcanica fa supporre una certa contemporaneità tra la deposizione di questi materiali e l'inizio dell'attività vulcanica della regione laziale.

$\Lambda 1$ disopra delle sabbie grialle a macrofossili vi è un livello di sabbia molto arrossata di circa 1 metro di potenza sul quale poggia un piccolo livello torboso che rappresenta l'inizio di una facies continentale che si svilupperà poi con la messa in posto dei depositi vulcanici sovrastanti.

Isa serie prosegue con un complesso di tufi "antichi ", costituiti, dal basso verso l'alto, da un livello di materiale pomiceo di circa 1 metro, da uno strato di 2 metri di sabbia gialla con abbondanti inclusi vulcanici (scorie), da uno strato di tufo granulare litoide grigio tipo "peperino" di 2 metri di potenza, e finalmente dalle pozzolane inferiori rosse con una potenza di oltre 4 metri.

A copertura dei sovraindicati materiali vi sono infine le sabbie rosse ferrettizzate, di trasporto eolico, che assumono potenza variabile tra i 2 ed i 5 metri.

Dalla sezione di Fig. 3, quindi, risulta evidente l'esistenza di una lacuna nella successione stratigrafica dei sedimenti, che si estende dal Pliocene Medio Superiore al tardo Quaternario; sono assenti i depositi del tardo Pliocene, del Calabriano e del Siciliano, che invece si riscontrano a circa $2 \mathrm{~km}$ in linea d'aria a Sud della cava di Pratica di Mare, nella serie stratigrafica regolare e continua, visibile in località "Campo Selva" a q. $37 \mathrm{~m}$ s.l.m. nei pressi di Torvajanica. 
Infatti in un'indagine eseguita nella località "Campo Selva " è stato possibile osservare i seguenti materiali descritti qui di seguito clall'alto verso il basso:

- una copertura di sabbia rossa ferrettizzata contenente limo e ghiaietto siliceo - Potenza massima 5 metri;

- esteso banco di argilla grigio-azzurra, ad elevata consistenza, stratificata ed in giacitura sub-orizzontale - Potenza massima 6 metri. Appartiene al Calabriano inferiore (zona a Hyalinea balthica) e l'ambiente di deposizione è marino, neritico medio;

- un deposito costituito da sabbia sciolta e cementata, ricco di resti di molluschi, riferibile all'intervallo Pliocene superiore-Calabriano inferiore, senza possibilità di ulteriori precisazioni ma che indica un ambiente di deposizione marino, neritico medio - Potenza massima 2 metri;

- infine un affioramento di argilla di colore grigio-azzurro, molto plastica ed a frazione granulometria minuta ed omogenea. L'età di questa argilla è del Pliocene superiore (parte alta); l'ambiente di deposizione è marino, neritico medio.

La lacuna stratigrafica precedentemente menzionata sembra interessare soprattutto la zona che da Pratica di Mare si estende verso Nord. Questo aspetto stratigrafico si ritrova anche ponendo in relazione alcuni sondaggi, eseguiti nella zona $(*)$.

Nella stratigrafia del pozzo G6, prossimo alla cava di Pratica di Mare, mancano i terreni del Calabriano; mentre ai pozzi G1-G2Pomezia 1 - Pomezia 2, si riscontra una continuità di sedimentazione dal Pliocene al Tirreniano.

Isa datazione sul Pliocene riportata sui sondaggi non concorda con la suddivisione da noi stabilita, in quanto da parte nostra si è fatto riferimento ai recenti studi e congressi sul Pliocene; tuttavia i medesimi sondaggi ci hanno permesso di ottenere maggiori ragguagli riguardo l'andamento paleo-morfologico e stratigrafico del basamento argilloso Pliocenico-Calabriano e di stabilire, con sufficiente approssimazione, il territorio maggiormente interessato dal fenomeno erosivo.

(*) Veri in "Indagini sulle acque sotterranee dell'Agro Romano e Pontino " - Parte I - Agro Romano. Sondaggi indicati con G1 - G2 - G6 Pomezia 1 - Pomezia 2. 
T)all'anclamento rlelle isobate rel tetto delle argille, riportate nell'opera sopracitata, si nota d'altra parte che il fenomeno erosivo si è impostato in una zona di massimo sollevamento; ne risulta pertanto che l'area oggetto del presente sturlio, è stata interessata da fenomeni tettonici che devono aver formato una serie di culminazioni e rlepressioni nell'ambito dei sedimenti argillosi, permettendo quindi all'erosione di agire con maggiore intensità sulle culminazioni più accentuate.

Dall:insieme delle consirlerazioni sopra esposte si può derlurre quindi che all'aspetto paleo-morfologico del sub-strato argilloso hanno contribuito fenomeni di carattere tettonico e conseguenti fenomeni di erosione concentrata; questi fattori, manifestatisi in grancle per tutto il territorio, hanno dato luogo, in particolare nella zona della cava di Pratica di Mare, a faglie e dislocazioni.

Allo scopo di ricostruire in rettaglio l'andamento paleo-morfologico del tetto del sub-strato argilloso circostante la zona della cava, si è attuata una campagna di prospezione geoelettrica.

I'indagrine geofisica è stata effettuata merliante l'esecuzione di undici sondaggi elettrici verticali disposti secondo profili opportunamente scelti (vedi Fig. 1) in modo da risolvere, nelle grandi linee, il problema dell'andamento e della profondità del tetto del sub-strato argilloso nella zona circostante la cava.

Le curve di resistività relative ai sondaggi elettrici vengono riportate nella tavola $I$.

I sondaggi sono stati effettuati con un'apparecchiatura potenziometrica a corrente continua ed è stata arlottata la disposizione elettrodica di Schlumberger-Sineriz con stese massime fra gli elettrorli di corrente $A B$ variabili, a secondo delle esigenze, tra i $40 \mathrm{~m} \mathrm{l}$. erl i $250 \mathrm{III} 1 .$.

L'interpretazione dei sondlaggi è stata eseguita merliante il confronto fra le curve di campagna e le curve teoriche; queste ultime sono state costruite utilizzando gli abachi rella C.G.G. di ArellanaMooney e gli abachi di Cagniard.

I sondaggi elettrici effettuati sui terreni in affioramento hanno consentito di attribuire agli elementi litologici della zona i valori della resistività sotto inclicati:

- Sabbie rosse e prorlotti piroclastici

- Sabbie calcaree con norluli di arenaria

- Iivelli argillosi; sabbie e ghiaie con limo

- Argille (plioceniche?)
$100 \mathrm{ohm} \cdot \mathrm{m}$

$40 \mathrm{ohm} \cdot \mathrm{m}$

$20 \mathrm{ohm} \cdot \mathrm{m}$

$15 \mathrm{ohm} \cdot \mathrm{m}$ 
'Tavola I - Curve di resistiviti.

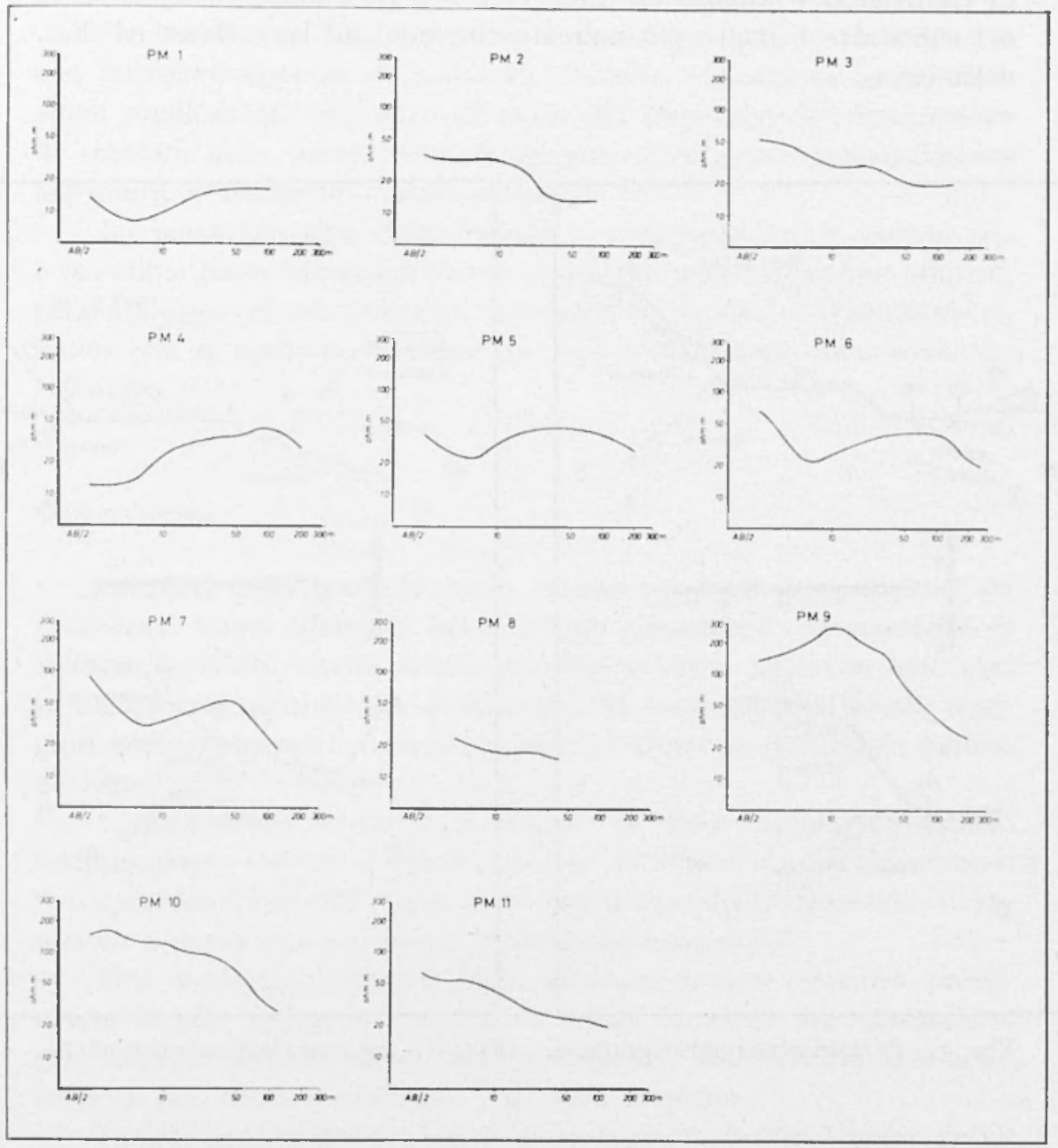

Dal confronto dei valori di resistività sopra riportati, relativi alle singole formazioni, con quelli ottenuti dai sondaggi elettrici effettuati in campagna, ̀̀ stato possibile ricostruire l'andamento dei vari mezzi geologici ed in particolare del tetto del sub-strato argilloso conduttivo (vedi Figg. 4,5).

L'analisi dei risultati ha permesso di fare le seguenti considerazioni.

È accertata l'esistenza di una continuità del tetto del sub-strato argilloso ed è confermato l'andamento paleo-morfologico dell'«alto " 
argilloso ad inclinazione differenziata, già riconosciuta con l'indagine di campagna; ì dimostrato che verso $\mathrm{N}$-NE, l'immersione del tetto del sub-strato ì molto più marcata che non sul lato Ovest ed Est, della cava.

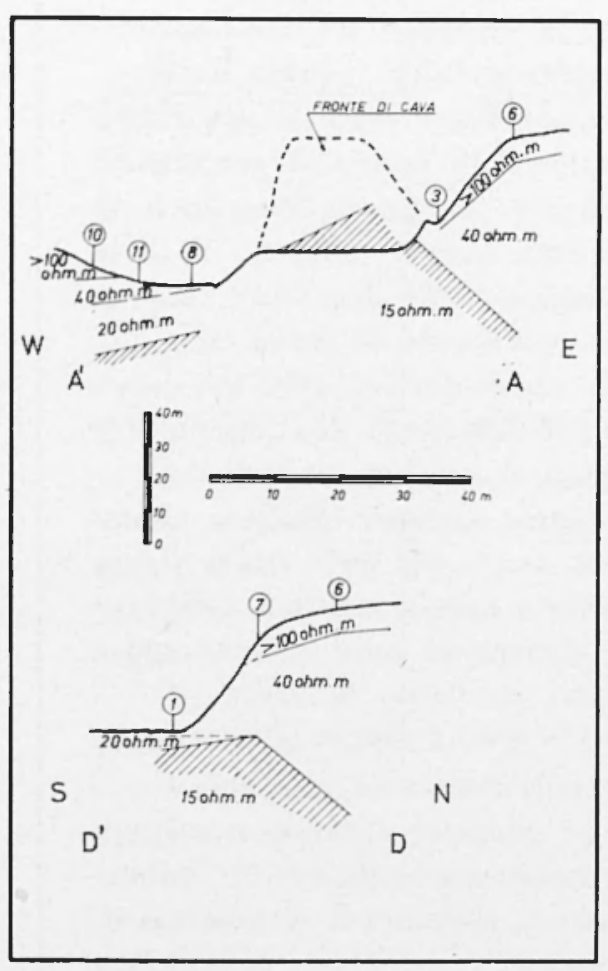

Fig. 4 - Sezioni elettrestratigrafiche.

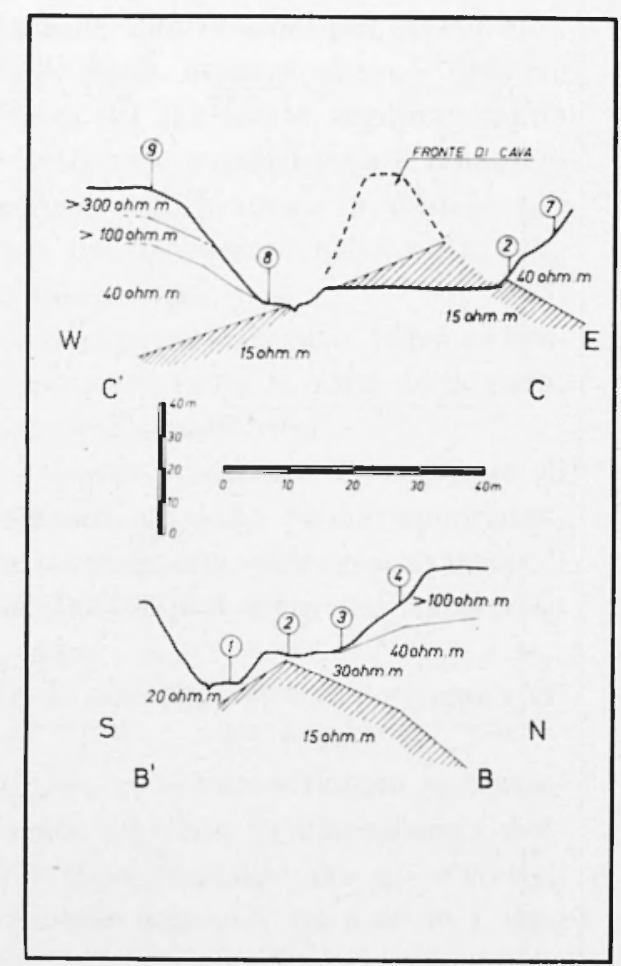

Fig. 5 - Sezioni elettrostratigrafiche.

Evidentemente l'attuale fronte di cava taglia, nella zona di massima curvatura, una culminazione del sub-strato argilloso, i cui fianchi presentano una immersione perimetrale ad andamento asimmetrico con valori particolarmente elevati di inclinazione nella parte orientale.

Analizzando i dati relativi alla coltre e confrontando insieme le quattro sezioni delle Figg. 1,5 si può osservare che l'elemento a resistività $40 \mathrm{ohm} \cdot \mathrm{m}$, rappresentato dalla formazione delle sabbie calcaree, poste alla base del piroclastico, subisce una notevole riduzione di spessore in direzione del Cimitero di Pratica di Mare; in tutte le altre 
sezioni questa formazione raggiunge spessori notevolmente maggiori come al sondaggio n. 6, ove risulta essere di circa 50 metri.

Evidentemente la deposizione delle sabbie calcaree d̀ avvenu1a con maggiore apporto di materiale nell'area circostante la culminazione argillosa gia esistente all'inizio del fenomeno; la diminuzione di spessore nella parte orientale, invece, deve essere principalmente attribuita a fenomeni erosivi locali.

La parte più alta della copertura, comprendente il piroclastico e le sabbie rosse ferrettizzate, con resistività superiore ai $100 \mathrm{ohm} \cdot \mathrm{m}$, presenta spessori relativamente uniformi, variabili fra i 10-20 metri, valori che si ripetono in quasi tutti gli affioramenti della zona circostante.

\section{Coxchtision}

Gli AA. della presente nota, considerati i risultati ottenuti da precedenti lavori eflettuati nel territorio circostante l'affioramento di Pratica di Mare, hanno rivolto l'indagine verso quegli aspetti che, in alcuni casi, permettono di ricavare, da consirlerazioni locali, maggiori conoscenze sui fenomeni generali avvenuti in particolari periodi geologici.

L'affioramento argilloso di Pratica di Mare rappresenta l'unica testimonianza visibile di quel processo tettonico che ha interessato il basamento Pliocenico-Calabriano prima che, nel Quaternario, si venissero a depositare i prodlotti sedimentari e vulcanici.

Una conferma indiretta della esistenza di una tettonica preesistente si può desumere osservando anche la carta del rilevamento gravimetrico eseguito dalla sezione Geofisica del Servizio Geologico d'Italia per tutto il territorio dell'Agro Romano.

Infatti analizzando le carte delle isoanomale del campo gravimetrico regionale della zona compresa fra Pomezia e Pratica di Mare, si osserva un accostarsi di anomalie positive e negative con rlisposizione antiappenninica e andamento decisamente parallelo e quasi rettilineo che indicano l'esistenza di una zona estremamente sollecitata; tale andamento limita a Nord la depressione di Ardea.

Anche le carte delle derivate prima e seconda del campo gravimetrico confermano la presenza di una zona sottoposta ad intense sollecitazioni le quali devono necessariamente aver interessato le parti profoncle del substrato e quelle superficiali. 
In particolare, nella zona in studio, la carta delle anomalie superficiali mostra la presenza di un minimo circonclato da una serie di valori positivi che indica la presenza di una zona di frattura.

Si cleduce pertanto, che la fascia compresa fra Pomezia e Pratica di Mare fa parte di quella importante zona rli frattura chiamata "Fagria di Arrlea n.

Anche landamento delle isobate del tetto delle argille, ricostruite merliante prospezioni elettriche dalla C.G.G. mostra che, nella zona a Nord rli Pratica di Mare, compresa fra Pomezia, Castel Romano e Solforata, il basamento plastico retermina un "alto paleo-morfologicoo" (alto rli Solforata) che porta le argille quasi in affioramento. Questo "altio ", costituendo il gradino più elevato che borda a Nord la depressione compresa tra Pomezia e Arlea (depressione Pomezia 1), raggiunge le quote più basse in rlirezione rlelle pendici occirlentali dell'apparato vulcanico laziale.

In base ai dati a disposizione è possibile riconoscere che le cause della depressione sono da ricercare sia nella tettonica che nella erosione, e poiché is preponderante l'azione del fenomeno tettonico su quello erosivo, si ritiene che la zona di raccordo fra l' «alto» di Solforata e la depressione di Pomezia 1, debba rappresentare l'area ove più intensi sono avvenuti sforzi e sollecitazioni; tale zona, proiettata sul piano orizzontale, può essere compresa in una fascia di terreno che ha come asse direzionale la congiungente Santa Palomba, Pomezia e Fosso di Pratica di Mare.

Quanto premesso trova conferma nel dettaglio e nelle osservazioni da noi fatte nella zona di Pratica di Mare.

L'affioramento argilloso rlella cava rappresenta a nostro avviso, una propagine dell' alto" di Solforata e la sua quota, insieme all'assetto paleo-morfologico circostante, inclica che trattasi di un "alto" relativo.

La disposizione attuale del banco argilloso di Pratica di Mare, in relazione con una tettonica a più vasto raggio appare ora decisamente più spiegabile in quanto la fascia di massime sollecitazioni conseguenti alle dislocazioni sembra comprendere decisamente la zona in studio. Trova cosi conferma la rlirettrice di faglia supposta lungo l'incisione Ic.

I'“alto " di Solforata, impostatosi principalmente con processi tettonici prequaternari, che lanno interessato l'intera regione formando una serie di culminazioni e rlepressioni nell'ambito dei serlimenti argillosi, ha subito frequenti e successivi fenomeni erosivi prima 
della deposizione della coltre sedimentaria e piroclastica del tardo Quaternario.

I processi erosivi, espletatisi necessariamente in maniera non uniforme, in conseguenza di una paleo-morfologia preesistente, laanno assunto localmente carattere di erosione concentrata, permettenclo, come all'affioramento di Pratica di Mare, di assumere un aspetto cupoliforme rissimmetrico su cui si è successivamente rlepositata, con giacitura sub-orizzontale, una litologia quaternaria di facies continentiale.

\section{BIBIIOGRAFIA}

(1) AMadei G., SBgre A. G., Tribotwo (i. - Aleune considerazioni sulla gravimetria e sulla geologia del ig. 15s, Lalina "Centro Geologico ¿'Italia", Servizio Geologico.

(2) Blaxc A. C., Conforto B., Ricco D., SAFA II., 1961. - Indagine sulle acque sotterranee dell Agro Romano e Pontino. Roma.

$\left.{ }^{3}\right)$ Conforto B., 1955. - Contributo alla conoscenza idrologiea della fascia costiera Laziale. "Geotecnica", 6. Roma.

(4) Fornaseri M., Scinerilio A., Ventriglia li., 1963. - La regione vulcanica dei Golli Albani (Vulcano Laziale). Centro di Mineralogria e Petrografia, Lniversita di Roma, C.N.R.

$\left.{ }^{5}\right)$ MotTa S., 1955. - Appunti preliminari sui rilevamenti geologici efletluati durante il 19.5.t nel fg. 158, Iatina. "Boll. Soc. Geol. Ital. ", 76, Roma.

(6) Segre A., 1957. - Note sui rilevamenti eseguili nel fg. 158, Latina, Carta Geologica d'Italia. "Boll. Serv. Geol.", 78. 